\title{
THE PHYSICAL NATURE OF WR8 (WN6/WC4)
}

\author{
PAUL A. CROWTHER, LINDA J. SMITH and ALLAN J. WILLIS \\ Department of Physics and Astronomy, University College London \\ Gower Street, London WC1E 6BT, UK
}

\begin{abstract}
An analysis of the UV, optical and IR spectra of the composite WN/WC star WR8 is presented using the WR standard model. All spectral features are consistent with formation in the same stellar wind. The stellar parameters are $T_{*}=49 \mathrm{kK}, \log L / L_{\odot}=5.1$, $\log \dot{M}=-4.2, v_{\infty}=1590 \mathrm{~km} \mathrm{~s}^{-1}$. The derived chemistry $(\mathrm{C} / \mathrm{He}=0.025, \mathrm{C} / \mathrm{N}=3, \mathrm{C} / \mathrm{O}=4)$ is intermediate between normal WN and WC stars.
\end{abstract}

Key words: stars:atmospheres - Wolf-Rayet - abundances - winds - mass-loss

\section{Introduction}

Conti \& Massey (1989) assigned WN/WC classifications to 8 Galactic and LMC Wolf-Rayet stars which showed anomalously strong C IV $\lambda 5801-12$ relative to $\mathrm{He}$ II $\lambda 4686$ in otherwise normal WN stars. The identification and analysis of composite $\mathrm{WN} / \mathrm{WC}$ stars, as opposed to $\mathrm{WN}+\mathrm{WC}$ binaries, have important consequences on single star evolutionary models. Recently, conflicting arguments about the nature of one such star, WR8 = HD 62910 (WN6/WC4), have been put forward. Radial velocity measurements by Niemela (1991) suggest that the nitrogen emission lines marginally move in anti-phase with the carbon spectrum implying a WN+WC system while an analysis by Willis \& Stickland (1990) concluded that the carbon and nitrogen emission lines were formed in the same wind. We present a detailed study of this star in an attempt to determine its true physical status.

\section{Observations and technique}

We use the WR standard model of Hillier (1990) to determine the physical parameters of WR 8 assuming it to be single, using UV and optical observations from Willis \& Stickland (1990) and new UKIRT-CGS4 spectra covering $1.6-2.3 \mu \mathrm{m}$. Since the carbon content of WR8 is non-negligible, our analysis requires the simultaneous determination of the physical parameters $\left(T_{*}, \log L_{*}, \dot{M}\right)$ and carbon abundance of WR8 using diagnostic helium (He I $\lambda 5876$, He II $\lambda 5411$ ) and carbon (C III $\left.\lambda 5696, \mathrm{C}_{\text {IV }} \lambda 5471\right)$ lines. We determine an interstellar reddening of $\mathrm{E}_{b-v}=0.60$ towards WR8 implying $\mathrm{M}_{v}=-4.5$ using a distance of $3.4 \mathrm{kpc}$ (Lundström \& Stenholm 1984). Once the temperature structure has been determined, nitrogen and oxygen abundances are then derived. 


\section{Results and evolutionary status}

Our analysis demonstrates that all UV, optical and IR spectral features can be simultaneously fitted to within a factor of 2-3 indicating that they are consistent with formation in a single stellar wind. The derived parameters for WR8 are somewhat different from previous Sobolev (Willis \& Stickland 1990) and pure helium standard model (Schmutz et al. 1989) analyses indicating that it is essential to perform standard model analyses including CNO elements for such stars. We compare the derived physical parameters and chemistry of WR 8 with a WN6 and a WC5 star in Table I. The elemental abundances of WR8 are indeed intermediate between $\mathrm{WN}$ and WC subtypes implying that WR 8 is a genuine transition WN/WC object. Our results broadly support the evolutionary predictions of Langer (1991) for WN/WC stars, although the increase in carbon at the expense of nitrogen appears to be more gradual and oxygen increases more rapidly than predicted. Finally, we note that the WN6/WC4 subtype of this star is misleading since the principal oxygen line spectrum of WR8 is that of O III-IV. Therefore the WC4 classification results from $\mathrm{O}$ III $\lambda 5592>\mathrm{C}$ III $\lambda 5696$, instead of the usual $\mathrm{O} v \lambda 5590$ feature with the weakness of $\mathrm{C}$ III attributable to the low carbon content for WR8 compared with normal WC stars.

TABLE I

Comparison of WR8 with a WN6 (Crowther 1993) and WC5 star (Hillier 1989)

\begin{tabular}{llcccrrrrr}
\hline Star & Subtype & $\begin{array}{c}T_{*} \\
\mathrm{kK}\end{array}$ & $\begin{array}{c}\log L_{\odot} \\
\mathrm{L}_{\odot}\end{array}$ & $\begin{array}{r}\log \dot{M} \\
\mathrm{M}_{\odot} / \mathrm{yr}\end{array}$ & $\begin{array}{r}v_{\infty} \\
\mathrm{km} / \mathrm{s}\end{array}$ & $\begin{array}{r}\log \\
\mathrm{H} / \mathrm{He}\end{array}$ & $\begin{array}{r}\log \\
\mathrm{C} / \mathrm{N}\end{array}$ & $\begin{array}{r}\log \\
\mathrm{C} / \mathrm{He}\end{array}$ & $\begin{array}{r}\log \\
\mathrm{O} / \mathrm{He}\end{array}$ \\
\hline WR134 & WN6 & 61 & 5.3 & -3.9 & 1950 & $<-2.0$ & -1.5 & -4.0 & -4.0 \\
WR8 & WN6/C4 & 49 & 5.1 & -4.2 & 1590 & $<-2.0$ & +0.5 & -1.7 & -2.3 \\
WR111 & WC5 & 59 & 5.0 & -4.4 & 1800 & $<-2.0$ & $\gg 2$ & -0.3 & -1.0 \\
\hline
\end{tabular}

\section{References}

Conti, P.S., Massey, P. 1989, ApJ 337, 251

Crowther, P.A. 1993, PhD thesis, University of London

Hillier, D.J. 1989, $A p J$ 347, 392

Hillier, D.J. 1990, $A \& A$ 231, 116

Langer, N. 1991, $A \mathscr{E} A$ 248, 531

Lundström, I., Stenholm, B. 1984, A\&A Suppl. 58, 163

Niemela, V.S. 1991, in: K.A. van der Hucht \& B. Hidayat, B. (eds.), Wolf-Rayet Stars and Interrelations with other Massive Stars in Galaxies, Proc. IAU Symp. No. 143 (Dordrecht: Kluwer), p. 201

Schmutz, W., Hamann, W-R., Wessolowski, U. 1989, $A \& A$ 210, 236

Willis, A.J., Stickland, D.J. 1990, $A \mathscr{E} A$ 232, 89 